\title{
Chaotic Behaviors in a Nonlinear Game of Two-Level Green Supply Chain with Government Subsidies
}

\author{
Chang-Feng Zhu' ${ }^{1}$ and Qing-Rong Wang $\mathbb{D}^{2}$ \\ ${ }^{1}$ School of Traffic and Transportation, Lanzhou Jiaotong University, Lanzhou, Gansu 730070, China \\ ${ }^{2}$ School of Electronic and Information Engineering, Lanzhou Jiaotong University, Lanzhou, Gansu 730070, China \\ Correspondence should be addressed to Qing-Rong Wang; wangqr@mail.lzjtu.cn
}

Received 12 October 2020; Revised 27 November 2020; Accepted 3 December 2020; Published 15 December 2020

Academic Editor: Y. T. Peter Bian

Copyright ( $) 2020$ Chang-Feng Zhu and Qing-Rong Wang. This is an open access article distributed under the Creative Commons Attribution License, which permits unrestricted use, distribution, and reproduction in any medium, provided the original work is properly cited.

\begin{abstract}
In this paper, a two-level green supply chain composed of a manufacturer and a retailer is taken as the background. Considering the consumer's double consumption preference and the manufacturer's green product R\&D investment, a differential game model of the green supply chain under the government cost subsidy strategy is constructed. Firstly, the equilibrium points of the system are solved and their stability is discussed and analyzed. Secondly, the dynamic evolution process of Nash equilibrium under the parameters of green degree, green preference coefficient, retail channel preference coefficient, coefficient of the sensitivity of price, and adjustment speed are described by numerical simulation. The results show that the two ways of a system entering chaos are Flip bifurcation and N-S bifurcation, respectively, by 2D bifurcation graph, and it is also verified in $1 \mathrm{D}$ bifurcation diagram. When the bifurcation parameters are small, the system maintains Nash equilibrium stability. If the green degree of products is increased, the green preference coefficient will also increase; on the contrary, the retail preference coefficient will decrease. Research and development cost subsidy policy can effectively improve the green degree of products and increase the sales volume of products, so as to improve the profit of supply chain members.
\end{abstract}

\section{Introduction}

In recent years, more and more attention has been paid to environmental protection in the world, and corresponding environmental protection laws and regulations have been formulated. On the other hand, consumers' awareness of environmental protection is also strengthening. These behaviors urge suppliers to implement green production, so as to reduce the environmental damage caused by people's production activities [1-3]. The concepts of green economy and ecological protection have been gradually and deeply rooted in the hearts of the people, which have changed the production and business model of enterprises. Green production capacity has become an important indicator for enterprises to survive in the industry for a long time. Manufacturing enterprises improve the quality of products by using green and environment-friendly materials. Green products can not only attract the attention of consumers, so as to expand product demand and improve product competitiveness, but also reduce environmental pollution and create a good social image [4-6]. The State Council has also encouraged the green production of enterprises, guided the green consumption of the masses, and proposed to build a green manufacturing system. However, when manufacturing enterprises are faced with many obstacles in the process of green production, such as insufficient funds, technological innovation, and insufficient support for product research and development, the enthusiasm of green product production is affected. Therefore, the government has formulated the corresponding green subsidy policy in order to subsidize the enterprises producing green products and promote the green development of the supply chain.

Many scholars have done in-depth research on the green supply chain from many angles and achieved satisfactory research results. Ghosh and shah [7] studied the secondary supply chain composed of a manufacturer and 
a retailer. The demand of the market is determined by the price of the product and the green degree of the product. The relationship between the price of the product and the green degree of the product depends on the pricing between them. Li et al. [8] built a green supply chain game model under price strategy based on Stackelberg game. The paper mainly analyzed the advantages and disadvantages of competition between decentralized decision and centralized decision-making. The research results showed that when the green cost exceeds a certain critical value, manufacturers will open direct sales channels, and the price of green supply chain products under the centralized strategy is higher than that under decentralized decision-making. Literature [9-11] discussed and analyzed the impact of consumers' green preference and environmental protection awareness on green supply chain decision-making. Huang et al. $[2,12]$ considered the pricing decision of closed-loop supply chain with the green degree of products under the same preference, and they found that green degree and fair preference will change the retail price, wholesale price, and waste recovery rate of products and also affect the total profit of the supply chain. Many scholars also studied the green supply chain system under government intervention [4]. For example, Sheng and other scholars [13-20] studied the impact of government subsidies on enterprise innovation. Madani et al. [21-24] considered the game model under the situation of green consumption subsidies and nongreen taxation and analyzed and discussed the influence of government fiscal strategy on the optimal strategy of supply chain members under centralized and decentralized decision-making. In this paper, we consider increasing innovation subsidies and construct effective measures that can make innovation subsidies become incentives for enterprises to produce green products.

In conclusion, the existing literature on green supply chain mainly focused on the green degree of products, government subsidies, and preferential policies of supply chain members. Little literature established a green supply chain model based on consumers' double consumption preference, and few scholars studied the impact of product greenness and government subsidy policies on enterprise profits from the perspective of dynamics. Therefore, this paper introduces the green preference and channel preference into the green supply chain management under the government subsidy strategy and constructs the green supply chain differential game model under the Government R\&D cost subsidy. This paper discusses the impact of double consumption and government subsidy policy on product green degree, product price, and supply chain member profit.

\section{Establishment of Model}

Consider a green supply chain system consisting of a manufacturer and a retailer. The manufacturer sells through two ways: one is to use traditional channels for wholesale sale and the other is to sell directly online. After comprehensive consideration of market factors, the manufacturer decides that the direct selling price and wholesale price of the product are $p_{d}$ and $w$, respectively, and the retailer determines the retail price $p_{r}$ according to the manufacturer's decision information and market demand. In addition, in order to encourage manufacturers to produce green products, the government decided to take the R\&D cost subsidy strategy to intervene with manufacturers. In order to facilitate the solution and the establishment of the model, we make the following assumptions about the model:

(1) Manufacturers not only need to pay fixed production costs to produce green products but also need to purchase new equipment or technological innovation to increase additional investment in green products. We assume that the relationship between R\&D costs of green products produced by manufacturers and greenness $e(e>0)$ is $\left(k e^{2} / 2\right)$, where $k(k>0)$ is the $\mathrm{R} \& \mathrm{D}$ cost coefficient of green products. In addition, it is assumed that the unit cost of the product produced by the manufacturer is $c$.

(2) Suppose that consumers have a preference for both channel and green. By the description of the demand function in $[7,25]$, we assumed that the demand of the retail channel and direct channel is a linear function and the cross price elasticity coefficient is symmetric. Thus, the demand functions of retail channel and direct channel are, respectively, expressed as

$$
\begin{aligned}
& Q_{r}=\theta \xi-\alpha p_{r}+\beta p_{d}+\gamma e, \\
& Q_{d}=(1-\theta) \xi-\alpha p_{d}+\beta p_{r}+\gamma e .
\end{aligned}
$$

Among them, $\xi$ represents the potential market capacity, $\theta(0<\theta<1)$ represents the retail channel preference coefficient, $\gamma$ represents the green preference coefficient, $\alpha$ is the price sensitivity coefficient, $\beta$ is the cross price sensitivity coefficient, and there are $\alpha>\beta>0$. In order to better analyze the characteristics of the green supply chain, referring to [26], $\left(\gamma^{2} / k\right)$ is defined as the efficiency coefficient of product greening. The higher the value of $\left(\gamma^{2} / k\right)$ is, the more the public likes green products, or it can be interpreted as that products with the same green quality need to pay less research and development costs.

In order to encourage manufacturers to improve the green quality of their products, the government considers subsidy strategies for green products [7, 27]. The government subsidizes the R\&D cost of the manufacturer's products. Assuming that the R\&D cost subsidy coefficient is $\mathcal{\varepsilon}(0<\varepsilon<1)$, the government subsidy expenditure under the $\mathrm{R} \& \mathrm{D}$ cost subsidy strategy is $\left(\varepsilon k e^{2} / 2\right)$.

In order to ensure the theoretical and practical significance of the supply chain, the model needs to meet the following constraints: 


$$
\begin{gathered}
Q_{r}>0, \\
Q_{d}>0, \\
c<w<p_{r}, \\
c<w<p_{d} .
\end{gathered}
$$

Therefore, when the manufacturing enterprise considers the public's green preference and channel preference and the government subsidizes the manufacturer according to the product $R \& D$ cost, the profit functions of the retailer and the manufacturer are, respectively,

$$
\begin{aligned}
& \pi_{r}=\left(p_{r}-w\right) Q_{r}, \\
& \pi_{m}=(w-c) Q_{r}+\left(p_{d}-c\right) Q_{d}-(1-\varepsilon) \frac{k e^{2}}{2} .
\end{aligned}
$$

By substituting equations (1) and (2) into (4) and (5), the profit functions of retailers and manufacturers under the dual preference and government subsidy strategy are, respectively,

$$
\begin{aligned}
& \pi_{r}=\left(p_{r}-w\right)\left(\theta \xi-\alpha p_{r}+\beta p_{d}+\gamma e\right) \\
& \pi_{m}=(w-c)\left(\theta \xi-\alpha p_{r}+\beta p_{d}+\gamma e\right)+\left(p_{d}-c\right)\left[(1-\theta) \xi-\alpha p_{d}+\beta p_{r}+\gamma e\right]-(1-\varepsilon) \frac{k e^{2}}{2}
\end{aligned}
$$

By taking partial derivatives of equations (6) and (7) with respect to $p_{r}$ and $p_{d}$, respectively, we can obtain the optimal first-order condition for the profits of retailers and manufacturers under dual preferences as follows:

$$
\begin{aligned}
& \frac{\partial \pi_{r}}{\partial p_{r}}=\beta p_{d}-2 \alpha p_{r}+\theta \xi+\gamma e+w \alpha, \\
& \frac{\partial \pi_{m}}{\partial p_{d}}=\beta p_{r}-2 \alpha p_{d}+(1-\theta) \xi+\gamma e+\beta w+c(\alpha-\beta) .
\end{aligned}
$$

If both the retailer and the manufacturer are bounded rational, that is, the two sides of the game do not fully understand each other's relevant information. Then, they determine the price of products in period $t$ according to the local estimation of the marginal target $\left(\partial \pi_{i} / \partial p_{i}\right)(i=r, d)$. In other words, in $t$ period, if $\left(\partial \pi_{i} / \partial p_{i}\right)>0$, then the manufacturer and retailer will increase the product price in $t+1$ period; on the contrary, if $\left(\partial \pi_{i} / \partial p_{i}\right)<0$, then the manufacturer and retailer will decrease the product price in $t+1$ period. According to this idea, the dynamic adjustment mechanism $[28,29]$ is introduced as follows:

$$
p_{i}(t+1)=p_{i}(t)+v_{i} p_{i}(t) \frac{\partial \pi_{i}}{\partial p_{i}}
$$

Where $v_{i}>0, i=r, d$ is the price adjustment speed of the retailer and the manufacturer, respectively. Combining equations (8), (9), and (10), a two-dimensional nonlinear discrete difference equation is obtained as

$$
\left\{\begin{array}{l}
p_{r}(t+1)=p_{r}(t)+v_{r} p_{r}(t)\left[\beta p_{d}-2 \alpha p_{r}+\theta \xi+\gamma e+w \alpha\right], \\
p_{d}(t+1)=p_{d}(t)+v_{d} p_{d}(t)\left[\beta p_{r}-2 \alpha p_{d}+(1-\theta) \xi+\gamma e+\beta w+c(\alpha-\beta)\right] .
\end{array}\right.
$$

For the convenience of calculation, let $A=\theta \xi+\gamma e+w \alpha$ and $B=(1-\theta) \xi+\gamma e+\beta w+c(\alpha-\beta)$; then, equation (11) can be written in the following form:

$$
\left\{\begin{array}{l}
p_{r}(t+1)=p_{r}(t)+v_{r} p_{r}(t)\left[\beta p_{d}-2 \alpha p_{r}+A\right] \\
p_{d}(t+1)=p_{d}(t)+v_{d} p_{d}(t)\left[\beta p_{r}-2 \alpha p_{d}+B\right] .
\end{array}\right.
$$

\section{Stability Analysis of the Model}

Let $P_{i}(t+1)=P_{i}(t)(i=r, d)$; then, we could directly get four equilibrium solutions of mapping (12) as follows: $E_{0}(0,0), \quad E_{1}((A / 2 \alpha), 0), \quad E_{2}(0,(B / 2 \alpha)), \quad$ and $E_{*}\left(\left(2 \alpha A+\beta B / 4 \alpha^{2}-\beta^{2}\right),\left(2 \alpha B+\beta A / 4 \alpha^{2}-\beta^{2}\right)\right)$.

Notice that $E_{0}$ is the trial fixed point, $E_{1}$ and $E_{2}$ are boundary fixed points, and $E_{*}$ is the internal fixed point. In order to guarantee that all the equilibrium points in system
(12) have practical economic significance, the four equilibrium points are nonnegative. From the definition of parameters, we know $\theta(0<\theta<1), \alpha>\beta>0, \xi, \gamma, e>0$, and $w>c>0$. Therefore, we can get $A>0, B>0,2 \alpha+\beta>0$, and $2 \alpha-\beta>0$. Thus, it is concluded that all four equilibrium points are nonnegative, and all equilibrium points are of economic significance. For the sake of obtaining the global stable conditions of fixed points of mapping (12), then, the Jacobian matrix of mapping (12) at any point $\left(p_{r}, p_{n}\right)$ is given as follows:

$$
J\left(p_{r}, p_{d}\right)=\left(\begin{array}{cc}
1+v_{r}\left[A+\beta p_{d}-4 \alpha p_{r}\right] & v_{r} \beta p_{r} \\
v_{d} \beta p_{d} & 1+v_{d}\left[B+\beta p_{r}-4 \alpha p_{d}\right]
\end{array}\right) .
$$

Proposition 1. $E_{0}$ is an unstable source. 
Proof. The Jacobian matrix $J\left(E_{0}\right)$ of $E_{0}$ is

$$
J\left(E_{0}\right)=\left(\begin{array}{cc}
1+A v_{r} & 0 \\
0 & 1+B v_{d}
\end{array}\right) .
$$

Obviously, $J\left(E_{0}\right)$ is a diagonal matrix and eigenvalues of $J\left(E_{0}\right)$ are elements located on diagonal; that is, $\lambda_{1}=1+A v_{r}$ and $\lambda_{2}=1+B v_{d}$. Because of $v_{i}>0, i=r, d, A>0, B>0$, so $\lambda_{i}>1(i=1,2)$; thus, $E_{0}$ is an unstable node.

Proposition 2. When $0<v_{r}<(2 / A)$, the equilibrium point $E_{1}$ is a saddle point, and when $(2 / A)<v_{r}$, the equilibrium point $E_{1}$ is an unstable node.

Proof. By substituting equilibrium point $E_{1}$ into equation (13), the Jacobian matrix $J\left(E_{1}\right)$ of $E_{1}$ can be expressed as

$$
J\left(E_{1}\right)=\left(\begin{array}{cc}
1-A v_{r} & v_{r} \frac{\beta A}{2 \alpha} \\
0 & 1+v_{d}\left(B+\frac{\beta A}{2 \alpha}\right)
\end{array}\right) .
$$

The eigenvalues of matrix (15) are $\lambda_{1}=1-A v_{r}$ and $\lambda_{2}=1+v_{d}(B+(\beta A / 2 \alpha))$. From the definition of the previous parameters and proof, we can know that $v_{r}, v_{d}>0$, $\alpha>\beta>0$, and $A, B>0$. So, there is $\lambda_{2}>1$. When $0<v_{r}<(2 / A),\left|\lambda_{1}\right|<1$, equilibrium point $E_{1}$ is a saddle point; when $(2 / A)<v_{r},\left|\lambda_{1}\right|>1$, then $E_{1}$ is an unstable node.

Proposition 3. When $0<v_{d}<(2 / B)$, the equilibrium point $E_{2}$ is a saddle point; when $(2 / B)<v_{d}$, the equilibrium point $E_{2}$ is an unstable node.

In order to avoid redundancy, the proof process is no longer described.

Proposition 4. If and only if condition (19) holds, the Nash equilibrium is stable.

Proof. The Jacobian matrix $J\left(E_{*}\right)$ of $E_{*}$ is

$$
J\left(E_{*}\right)=\left(\begin{array}{cc}
1-v_{r} \frac{2 \alpha(2 \alpha A+\beta B)}{4 \alpha^{2}-\beta^{2}} & v_{r} \frac{\beta(2 \alpha A+\beta B)}{4 \alpha^{2}-\beta^{2}} \\
v_{d} \frac{\beta(2 \alpha B+\beta A)}{4 \alpha^{2}-\beta^{2}} & 1-v_{d} \frac{2 \alpha(2 \alpha B+\beta A)}{4 \alpha^{2}-\beta^{2}}
\end{array}\right) .
$$

The specific forms of trace $\operatorname{Tr}(J)$ and determinant $\operatorname{Det}(J)$ of $J\left(E_{*}\right)$ are given as

$$
\begin{aligned}
\operatorname{Tr}(J) & =2-v_{r} \frac{2 \alpha(2 \alpha A+\beta B)}{4 \alpha^{2}-\beta^{2}}-v_{d} \frac{2 \alpha(2 \alpha B+\beta A)}{4 \alpha^{2}-\beta^{2}}, \\
\operatorname{Det}(J) & =1-v_{d} \frac{2 \alpha(2 \alpha B+\beta A)}{4 \alpha^{2}-\beta^{2}}-v_{r} \frac{2 \alpha(2 \alpha A+\beta B)}{4 \alpha^{2}-\beta^{2}}+v_{r} v_{d} \frac{(2 \alpha A+\beta B)(2 \alpha B+\beta A)}{4 \alpha^{2}-\beta^{2}} .
\end{aligned}
$$

According to the Jury condition, the local stability conditions of the Nash equilibrium point $E_{*}$ are as follows:

$$
\left\{\begin{array}{l}
4-v_{r} \frac{4 \alpha(2 \alpha A+\beta B)}{4 \alpha^{2}-\beta^{2}}-v_{d} \frac{4 \alpha(2 \alpha B+\beta A)}{4 \alpha^{2}-\beta^{2}}+v_{r} v_{d} \frac{(2 \alpha A+\beta B)(2 \alpha B+\beta A)}{4 \alpha^{2}-\beta^{2}}>0, \\
v_{r} v_{d} \frac{(2 \alpha A+\beta B)(2 \alpha B+\beta A)}{4 \alpha^{2}-\beta^{2}}>0, \\
v_{r} \frac{2 \alpha(2 \alpha A+\beta B)}{4 \alpha^{2}-\beta^{2}}+v_{d} \frac{2 \alpha(2 \alpha B+\beta A)}{4 \alpha^{2}-\beta^{2}}-v_{r} v_{d} \frac{(2 \alpha A+\beta B)(2 \alpha B+\beta A)}{4 \alpha^{2}-\beta^{2}}>0 .
\end{array}\right.
$$

And by the definition of the parameters, we know $v_{r}, v_{d}>0, \alpha>\beta>0$, and $A, B>0$, so $2 \alpha-\beta>0$. Obviously, the second condition in the inequality system (18) holds. Then, inequality group (18) can be rewritten as 


$$
\left\{\begin{array}{l}
4\left(4 \alpha^{2}-\beta^{2}\right)-v_{r} 4 \alpha(2 \alpha A+\beta B)-v_{d} 4 \alpha(2 \alpha B+\beta A)+v_{r} v_{d}(2 \alpha A+\beta B)(2 \alpha B+\beta A)>0 \\
v_{r} 2 \alpha(2 \alpha A+\beta B)+v_{d} 2 \alpha(2 \alpha B+\beta A)-v_{r} v_{d}(2 \alpha A+\beta B)(2 \alpha B+\beta A)>0 .
\end{array}\right.
$$

Through the above analysis, the Nash equilibrium is stable if and only if the inequality set (19) is satisfied. Fix the initial value as $(0.6169,1.4878)$ and select parameters $\theta=0.4183, \xi=1.4896, \beta=0.0519, \alpha=0.597, \gamma=0.5171$, $e=0.4019, w=1.1462$, and $c=0.8133$. Under this set of parameters, the stable region of equilibrium point $E^{*}$ with respect to price adjustment speed $\left(v_{r}, v_{d}\right)$ is shown in the blue area in Figure 1. In this stable region, the prices $p_{d}$ and $p_{r}$ of manufacturer and retailer converge to the Nash equilibrium after many games. If the value of the adjustment speed $v_{r}$ or $v_{d}$ is increased, the point $\left(v_{r}, v_{d}\right)$ will jump out of the stable region, and complex dynamic phenomena will occur in the later process.

\section{Numerical Bifurcation Analysis}

As we all know, people mainly study the complex dynamic behavior of the nonlinear dynamic system by numerical simulation, and the tools used in the process of numerical simulation mainly include 1D bifurcation diagram, 2D bifurcation diagram, Lyapunov exponent diagram [30, 31], and phase diagram. In this section, the influence of the parameter changing on the stability of the Nash equilibrium of system (12) is discussed by numerical analysis.

4.1. The Influence of Price Sensitive Coefficient on the System. In order to study the effect of price sensitive coefficient $\alpha$ on the system, we select a group of parameters such as $\theta=0.4183, \xi=1.4896, \beta=0.0519, \gamma=0.5171, e=0.4019$, $w=1.1462$, and $c=0.8133$. When the value of the parameter $\alpha$ increases gradually, 2D bifurcation diagrams of system (12) regarding the adjusted speeds $v_{r}$ and $v_{d}$ are obtained, as shown in Figure 2. The different colors in the $2 \mathrm{D}$ bifurcation diagram represent different periods (i.e., the color displayed by the color bar on the right side of the bifurcation diagram). The brown region represents the Nash equilibrium stable region, namely, 1-period, light green represents 2-period, dark green represents 3-period, orange represents 4-period, and so on, black represents quasiperiodic state or chaos, and white area represents escape state. These three states cannot be calculated at present. The stable region of the Nash equilibrium point with respect to adjustment speeds $v_{r}$ and $v_{d}$ is shown in the brown area in Figure 2. In the parameter plane $\left(v_{r}, v_{d}\right)$, the manufacturer's direct selling price $p_{d}$ and retailer's selling price $p_{r}$ will converge to the Nash equilibrium point $E_{*}$ after many games. If we increase the value of $v_{r}$ or $v_{d}$, it will cause $v_{r}$ or $v_{d}$ to jump out of the stable region. From Figures 2(a), 2(b), 2(c), and 2(d), we observe that the system has two different paths into chaos. One is that, with the increase of parameter values $v_{r}$ and $v_{d}$, system (12) successively passes through the brown region (1-cycle), green region (2-cycle), orange region (4-cycle), light green region (8-cycle), and finally enters the black region; in other words, the system enters into the chaotic state by Flip bifurcation. When manufacturers and retailers determine their respective adjustment speed according to the above path, the system will enter into chaos through a period-doubling, which also means that a supply chain system composed of manufacturers and retailers will end up in chaos. With the increasing speed of adjustment, manufacturer or retailer may not survive and the supply chain will collapse. The other is that when the parameters $\left(v_{r}, v_{d}\right)$ enter the black region from the brown region (1cycle) through the green region (2 cycles), the system enters into quasiperiod and finally enters chaos through the Neimark-Sacker bifurcation. From these four pictures, we find that the path of the system into chaos is the same. The different value of price sensitivity coefficient $\alpha$ makes the unique graph of the score fork, and we also feel the complex dynamic phenomenon. Combined with the $2 \mathrm{D}$ bifurcation diagram and the above analysis, it is found that the price sensitive coefficient $\alpha$ will not affect the bifurcation way of the system, but will change the dynamic phenomenon of the fork. At the same time, we find that, in order to make the manufacturer and retailer obtain larger profits, the value of adjustment speed should not be too large. Once the threshold value is exceeded, the system may collapse. Therefore, choosing a smaller adjustment speed can promote the stable development of the green supply chain system composed of manufacturer and retailer.

4.2. The Influence of Green Degree on Retail Channel Preference Coefficient and Green Preference Coefficient. In order to better analyze the influence of green degree on retail channel preference coefficient $\theta$ and green preference coefficient $\gamma$ on the system, we choose a set of parameter values as $v_{r}=0.8587, v_{d}=0.849, \xi=1.4896, \beta=0.0519, \alpha=0.597$, $w=1.1462, c=0.8133$, and $\theta=0.4183$. Taking $\gamma$ as the bifurcation variable, the $1 \mathrm{D}$ bifurcation diagrams and their corresponding maximum Lyapunov exponent are obtained as shown in Figures 3(a), 3(b), 3(c), and 3(d). When $e=0.3019$, the $1 \mathrm{D}$ bifurcation diagram as shown in Figure 3(a) is obtained. When $0<\gamma<2.007$, the system is in Nash equilibrium and steady state, which indicates that the green supply chain system develops steadily. When $\gamma \approx 2.007$, the system begins bifurcation. When $2.007<\gamma<3.198$, the system is in a periodic state. When $3.198<\gamma<3.489$, the system is in a chaotic state, which indicates that the green supply chain system composed of a manufacturer and a retailer is in chaotic competition. When $3.489<\gamma<4.5$, the system experiences three-time bifurcations from period to chaos, which indicates that the manufacturer and retailer experience three price games. Similarly, when $e=0.4019,1 \mathrm{D}$ bifurcation diagram is shown in Figure 3(c). When $0<\gamma<2.676$, the system is in Nash equilibrium and stable state. When $\gamma \approx 2.676$, the 


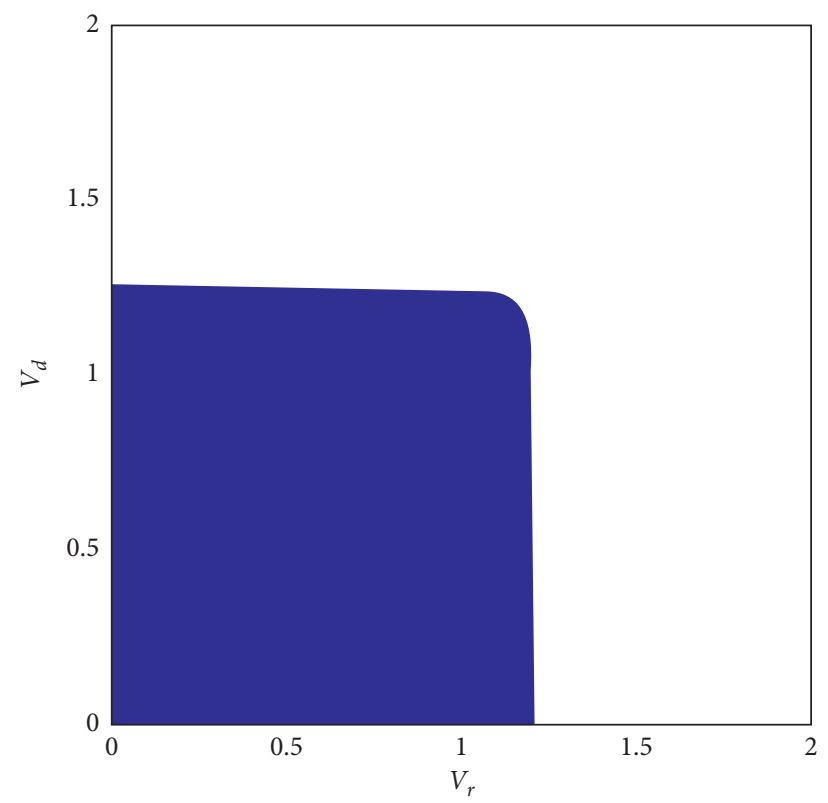

FIgURE 1: Local stable region of the equilibrium point $E^{*}$.

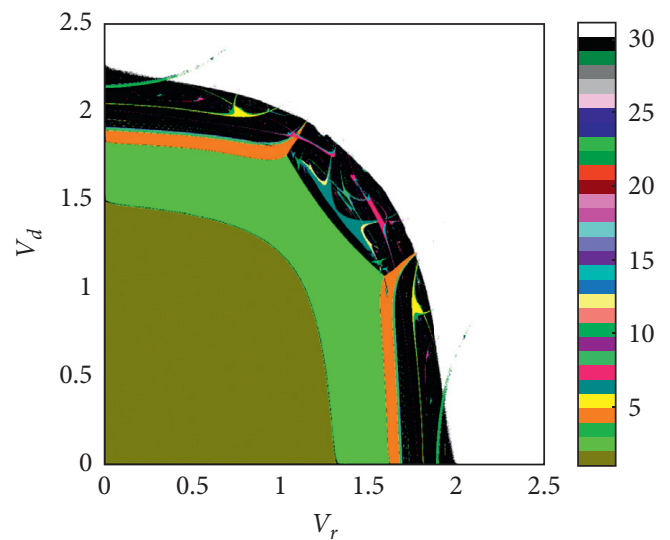

(a)

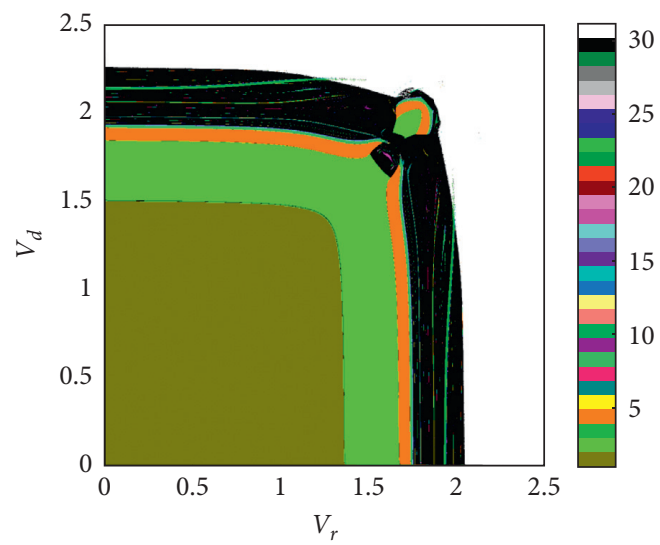

(c)

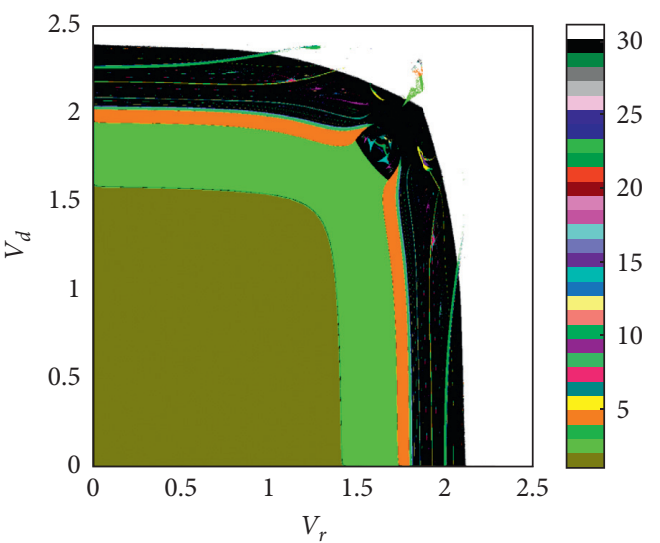

(b)

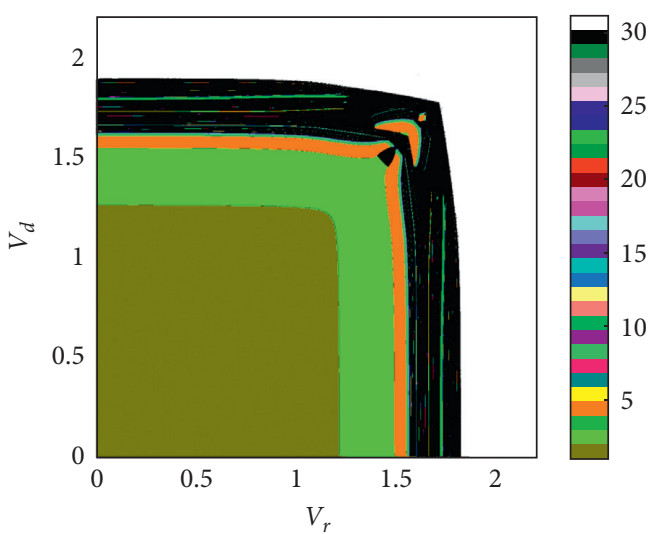

(d)

Figure 2: 2D bifurcation diagram of the system on the parameter plane $\left(v_{r}, v_{d}\right)$, where (a) $\alpha=0.097$, (b) $\alpha=0.237$, (c) $\alpha=0.337$, and (d) $\alpha=0.597$. 


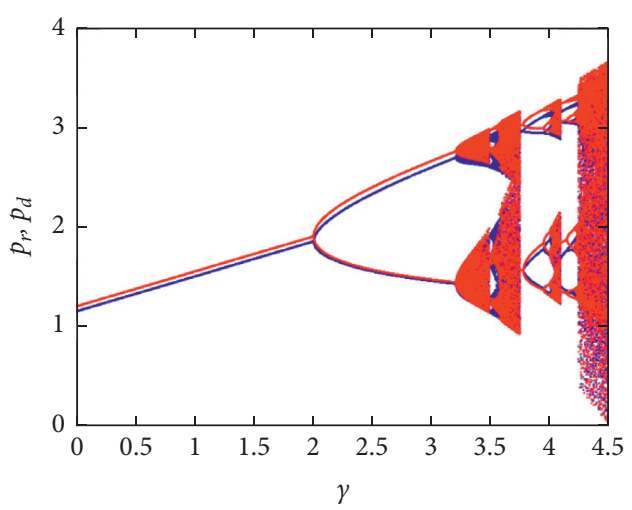

(a)

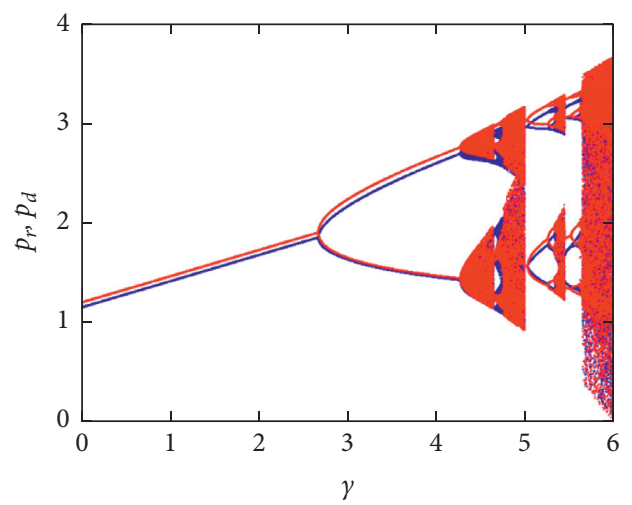

(c)

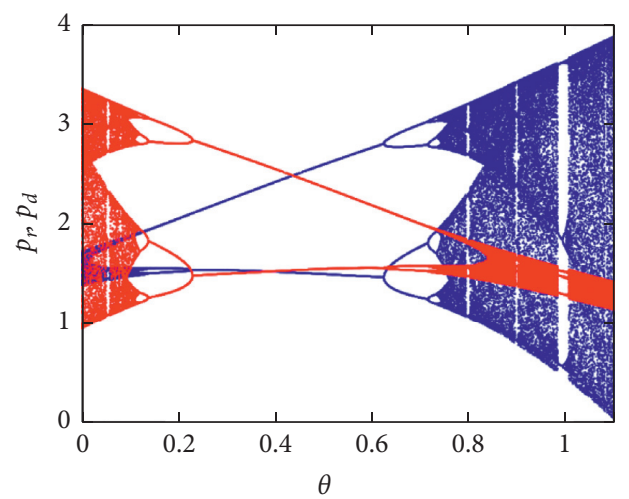

(e)

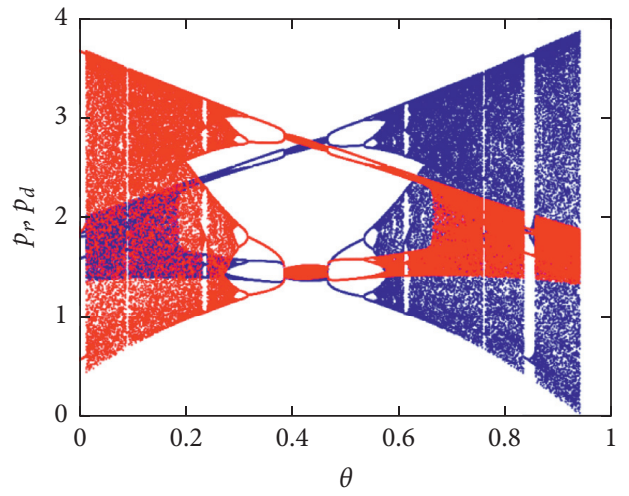

(g)

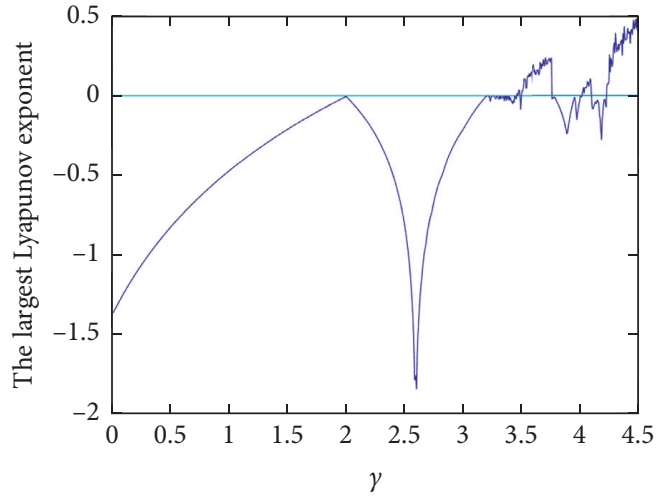

(b)

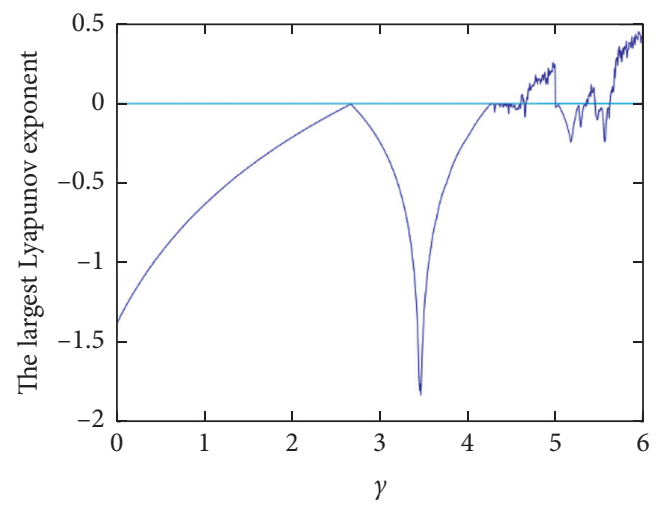

(d)

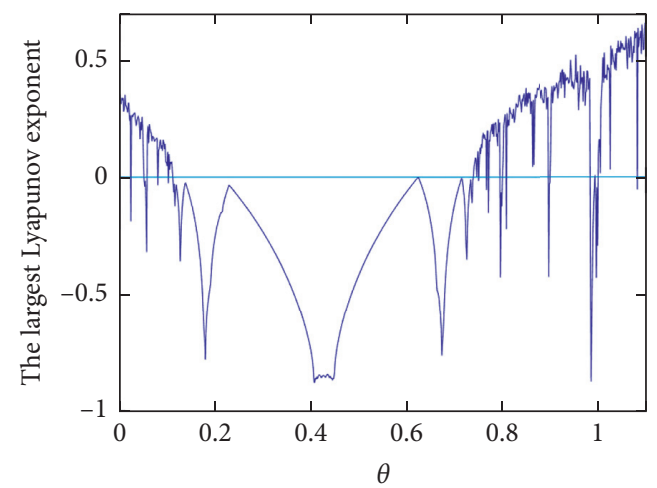

(f)

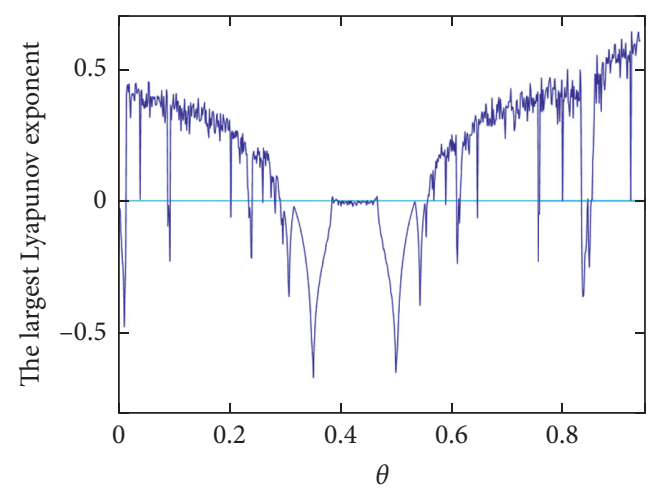

(h)

FiguRE 3: 1D bifurcation diagram with respect to green preference coefficient $\gamma$ and retail preference channel coefficient $\theta$ and their corresponding maximum Lyapunov exponent diagrams. Select a set of parameter values as $v_{r}=0.8587, v_{d}=0.849, \xi=1.4896, \beta=0.0519$, $\alpha=0.597, w=1.1462$, and $c=0.8133$, where (a) $e=0.3019$ and $\theta=0.4183$, (c) $e=0.4019$ and $\theta=0.4183$. Figures 3(b) and 3(d) are the maximum Lyapunov exponent diagrams corresponding to Figures 3(a) and 3(c), where (e) $e=2.1019$ and $\gamma=0.5171$, (g) $e=2.5019$ and $\gamma=0.5171$. Figures $3(\mathrm{f})$ and $3(\mathrm{~h})$ are the maximum Lyapunov exponent graphs corresponding to Figures 3(e) and 3(g). 
system bifurcates. When $2.676<\gamma<4.239$, the system is in a period state. When $4.239<\gamma<6$, the system alternates between chaos and periodic bifurcation. Figures 3(b) and 3(d) are the largest Lyapunov exponent graphs corresponding to Figures 3(a) and 3(c). Lyapunov exponent diagram is an important tool to study the nonlinear dynamic behavior. In the nonlinear dynamical system, the Lyapunov exponent graph describes the convergence or divergence of adjacent trajectories. It distinguishes the regular attractor from singular attractor. When the Lyapunov exponent is less than zero, we can judge that the attractor is a regular attractor. If at least one of the Lyapunov exponents is positive, then we can infer that there are singular attractors or chaotic attractors in the system. When $e=2.1019$ and $\mathrm{e}=2.5019$ (Figures 3(e) and 3(g) are obtained, resp.), it can be seen that the inverse N-S bifurcation occurs first in the system, followed by positive N-S bifurcation. It is obvious from these two diagrams that the stability interval of Figure $3(\mathrm{~g})$ is smaller than that of Figures 3(e), 3(f), and 3(h) which are the maximum Lyapunov exponent diagrams corresponding to Figures 3(e) and 3(g), respectively. The system experiences three-time bifurcation of entering chaos from the period, which indicates that the manufacturer and retailer experience three times of price game. When Lyp $<0$, it means that the system is in a stable state; when Lyp $=0$, it indicates that the system bifurcates at this point; when Lyp $>0$, the system enters into a chaotic state. It can be seen from the diagrams that when the green preference coefficient $\gamma$ is small and the value of the retail channel preference coefficient $\theta$ is in the middle value, the system will be in a stable state. At the same time, we also find that if the green degree of the product is increased, the green preference coefficient will also increase. On the contrary, when the green degree of the product increases, the value of the retail preference coefficient will decrease.

4.3. The Influence of Other Parameters on the System. In order to further analyze the influence of other parameters on system (12), we select $v_{r}, v_{d}, c, w, e$, and $\xi$, respectively, as the bifurcation variables and obtain the $1 \mathrm{D}$ bifurcation diagrams and the maximum Lyapunov exponent diagrams, as shown in Figures 4. In Figures 4(a) and 4(b), we can see that the internal equilibrium point $E_{*}$ is stable when the value of parameters $v_{r}$ and $v_{d}$ is relatively small. However, as the value of parameter $v_{r}$ continues to increase, the internal equilibrium $E_{*}$ is no longer stable. Suddenly, irregular dynamic behaviors appear, including N-S bifurcation, periodic window, and chaos. In Figure 4(b), the system experiences 2-period, 4-period, 2-period, and finally enters chaos; that is, the system enters chaos through Flip bifurcation. It can be seen from Figures 4(c), 4(d), and 4(f) that the stable 2-periodic loop loses its stability through the N-S bifurcation, and the system finally enters chaos. In Figure 4(e), the bifurcation diagram with respect to the green degree $\theta$ is depicted. We can see that the period-doubling bifurcation and N-S bifurcation appear simultaneously in the process of bifurcation. In addition, we also find that periodic windows appear in the process of bifurcation in Figures 3(c), 3(d), 3(e), and 3(f). In fact, the periodic window corresponds to the part of the Lyapunov exponent closed to zero. When the Lyapunov exponent is equal to zero, the system bifurcates at this point; when the Lyapunov exponent is greater than zero, the system enters into a chaotic state. It is found that when the bifurcation parameters are small, the system is in a steady state. In this case, the supply chain system composed of a single manufacturer and a single retailer develops stably. At the same time, it is found that increasing the green degree of products can make the system more stable. However, due to the limitation of research and development costs, manufacturers will not increase the green degree of products to meet the green preference of consumers. In other words, increasing the green degree of products can improve the sales of products and the profits of supply chain members.

\section{Global Dynamics Analysis of the System}

People often pay attention to the final state of the system in nonlinear dynamics. The final state reflects the final state of the system. The final state can be expressed by the attractor. In the process of studying the nonlinear dynamic system, it is found that the change of parameters will cause variation in number and structure of attractors. Then, the influence of cross price sensitivity coefficient on the final behavior of system (12) is discussed through the evolution process of the attractor.

Parameters $\quad v_{r}=0.3584, \quad v_{d}=0.4005, \quad \theta=0.7568$, $\xi=1.079, \alpha=1.6181, \gamma=0.6934, e=0.5, w=2.4113$, and $c=1.5383$ are fixed and $\beta$ is taken as the variable to obtain the attractor evolution diagrams, as shown in Figure 5. When $\beta=0.5827$, a 2 -periodic attractor is observed in Figure $5(\mathrm{a})$. With the increase in the cross price sensitivity coefficient $\beta$, the 2-period attractor evolves into periodic cycles with rough edges as shown in Figure 5(b). When $\beta=0.6357$, the original 2 -period ring with rough edges recedes and gradually evolved into a larger 2 -period ring. When the value of parameter $\beta$ continues to increase, the attractor becomes larger and larger. Until $\beta=0.7247$, the 2period cycles break to form many very small attractors, which are called phase-locking, as shown in Figure 5(e). The broken attractors recombine to form two attractors which look like a "ring" as shown in Figure 5(f). It is found that the number and shape of attractors change significantly when the cross price sensitivity coefficient $\beta$ increases. When $\beta=0.7747$, two strange attractors joint 


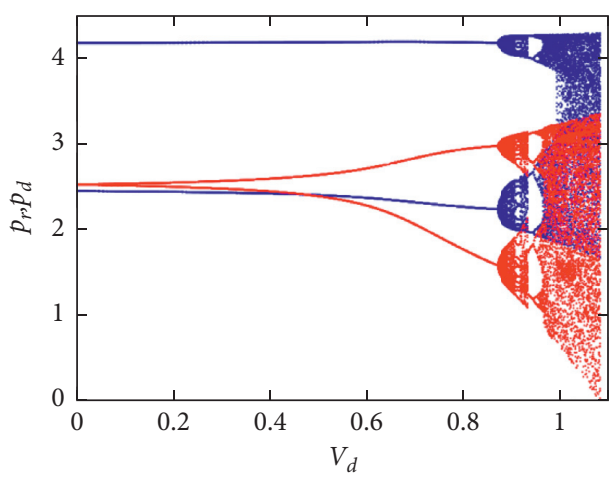

(a)

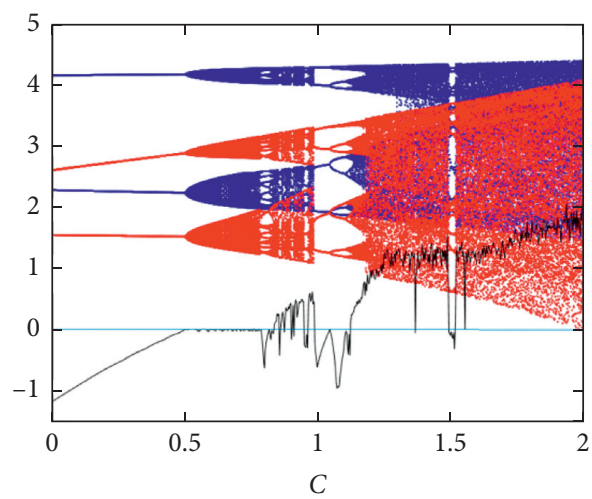

(c)

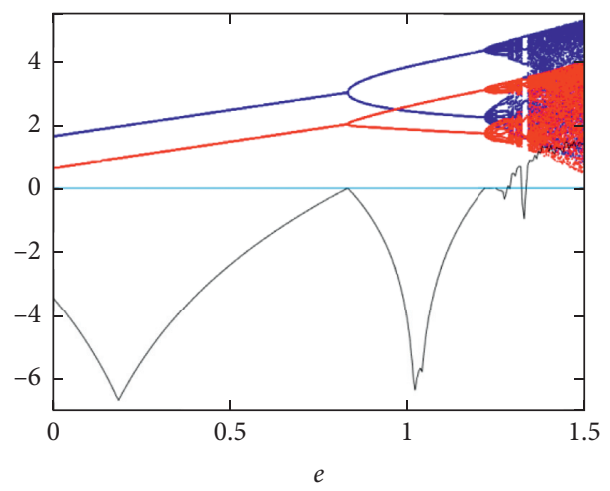

(e)

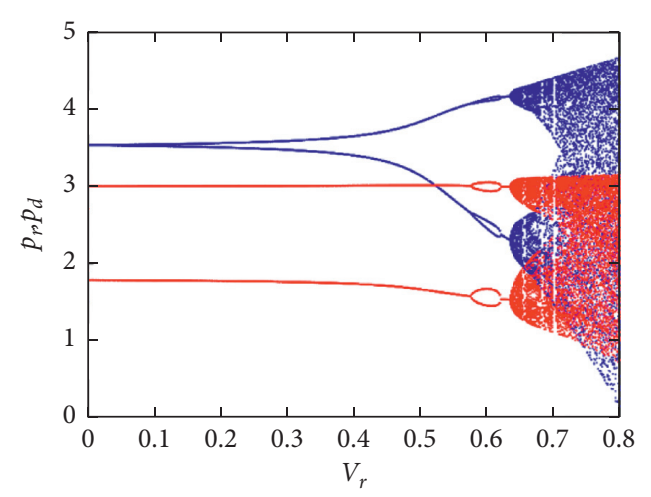

(b)

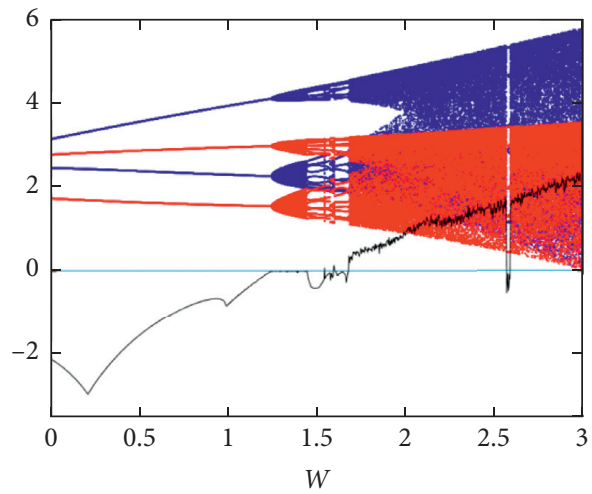

(d)

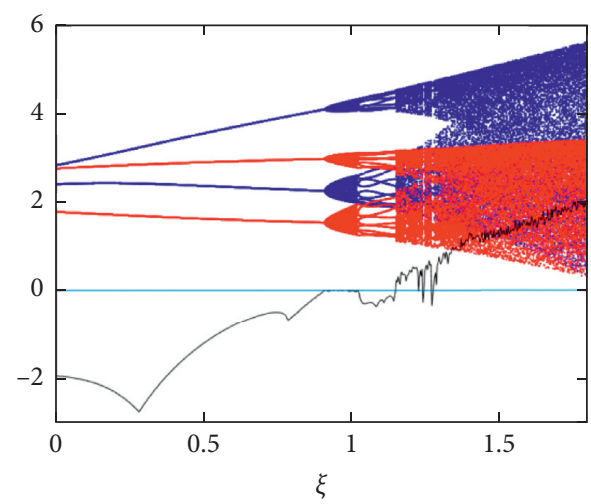

(f)

FiguRE 4: 1D bifurcation diagrams and maximum Lyapunov exponent diagrams of system (12) with fixed parameters are $\theta=0.9037$, $\beta=0.083, \alpha=0.4981$, and $\gamma=1.5192$. (a) Taking $v_{r}$ as a bifurcation parameter, the other parameters are $\xi=0.9805, v_{d}=0.8967, e=1.1555$, $c=0.6784$, and $w=1.3669$. (b) Taking $v_{d}$ as a bifurcation parameter, the other parameters are $v_{r}=0.6489, \xi=0.9805, e=1.1555$, $c=0.6784$, and $w=1.3669$. (c) Taking $c$ as bifurcation parameter, the other parameters are $v_{r}=0.6489, v_{d}=0.8967, \xi=0.9805, e=1.1555$ and $w=1.3669$. (d) Taking $w$ as a bifurcation parameter, the other parameters are $v_{r}=0.6489, v_{d}=0.8967, \xi=0.9805, e=1.1555$, and $c=0.6784$. (e) Taking $e$ as a bifurcation parameter, the other parameters are $v_{r}=0.6489, v_{d}=0.8967, \xi=0.9805, w=1.3669$, and $c=0.6784$. (f) Taking $\xi$ as a bifurcation parameter, the other parameters are $v_{r}=0.6489, v_{d}=0.8967, e=1.1555, w=1.3669$, and $c=0.6784$.

together, as shown in Figure 5(g). With the further increase of the parameter $\beta$, two interlocking chaotic attractors evolve into a chaotic attractor as shown in Figure 5(h). Finally, the system is in a chaotic state, which means that the supply chain composed of a manufacturer and a retailer is in a chaotic competition scenario. Therefore, it shows that increasing the value of cross price sensitivity coefficient $\beta$ will make the market more and more chaotic, which is against the win-win intention of manufacturer and retailer. Therefore, choosing a smaller cross price sensitivity coefficient is conducive to the common development of manufacturers and retailers. 


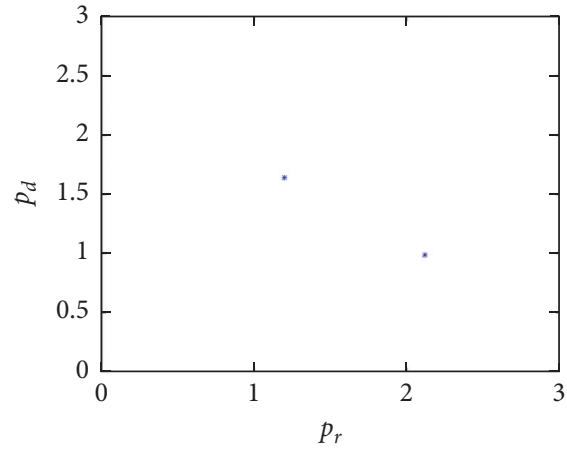

(a)

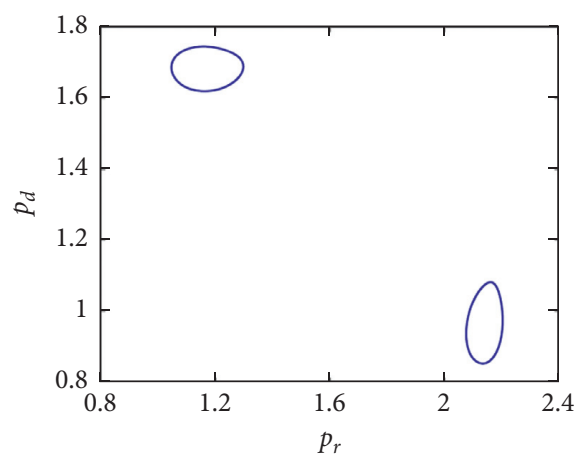

(c)

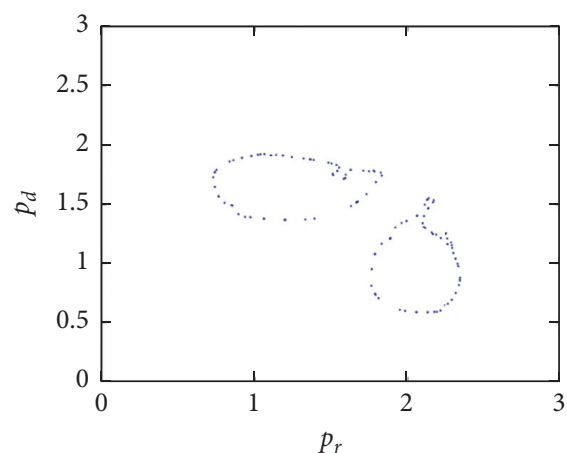

(e)

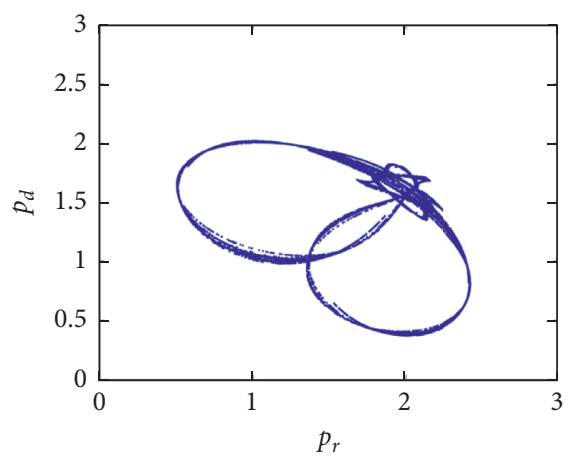

(g)

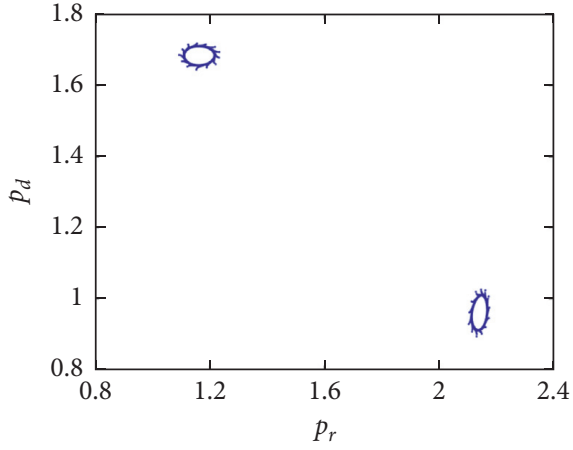

(b)

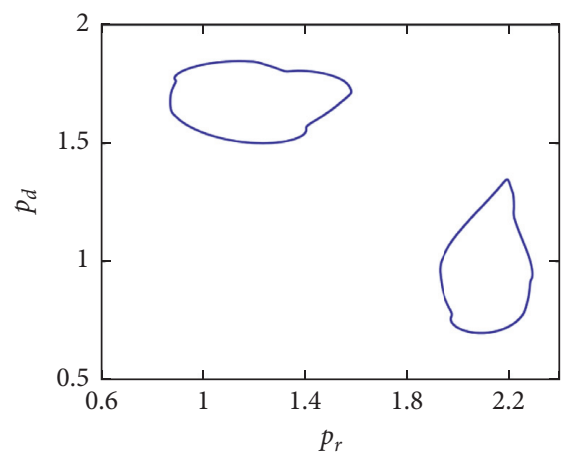

(d)

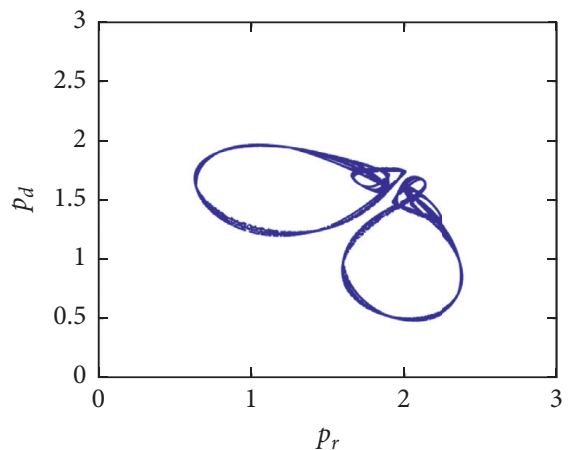

(f)

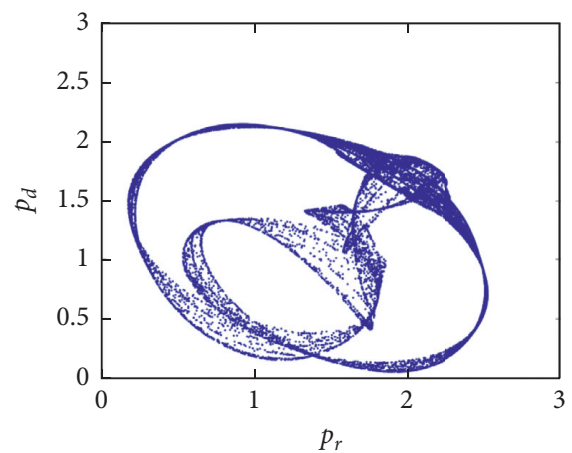

(h)

Figure 5: Diagrams of attractors evolution. (a) $\beta=0.5827$, (b) $\beta=0.6277$, (c) $\beta=0.6357$, (d) $\beta=0.6887$, (e) $\beta=0.7247$, (f) $\beta=0.7487$, (g) $\beta=0.7747$, and (h) $\beta=0.8327$. 


\section{Conclusion}

In this paper, a two-level green supply chain composed of a single manufacturer and a single retailer is taken as the background. Firstly, considering the consumer's double consumption preference and the manufacturer's green product $R \& D$ investment, the game model of the green supply chain under the government cost subsidy strategy is constructed. Secondly, the dynamic evolution process of Nash equilibrium under the parameters of green degree, green preference coefficient, retail channel preference coefficient, coefficient of sensitivity of price, and adjustment speed is described by numerical simulation. The results show that the two ways of the system entering into chaos are Flip bifurcation and N-S bifurcation. The N-S bifurcation is mainly discussed in this paper. When the bifurcation parameters are small, the system maintains Nash equilibrium stability, and the stability of the system is destroyed if it exceeds a certain threshold value. Increasing the green degree of the product will increase the green preference coefficient, but the retail preference coefficient will decrease. The subsidy policy of R\&D cost plays an irreplaceable role in encouraging the manufacturer to produce green products. Green sales can effectively improve the sales volume of products and realize the win-win benefits between manufacturers and retailers.

\section{Data Availability}

No data were used to support this study.

\section{Conflicts of Interest}

The authors declare that the research was conducted in the absence of any conflicts of interest.

\section{Acknowledgments}

This research was supported by the National Natural Science Foundation of China (Grant no. 71961016) and the Foundation of A Hundred Youth Talents Training Program of Lanzhou Jiaotong University.

\section{References}

[1] Q. Zhu, J. Sarkis, and K.-H. Lai, "Institutional-based antecedents and performance outcomes of internal and external green supply chain management practices," Journal of Purchasing and Supply Management, vol. 19, no. 2, pp. 106-117, 2013.

[2] Z.-Y. Zhang, D.-X. Fu, and Q. Zhou, "Optimal decisions of a green supply chain under the joint action of fairness preference and subsidy to the manufacturer," Discrete Dynamics in Nature and Society, vol. 2020, Article ID 9610503, 18 pages, 2020.

[3] J. Li, Y. Yi, and H. Yang, "Information investment and sharing in a two-echelon supply chain under government subsidy and consumer preference for energy-saving products," Mathematical Problems in Engineering, vol. 2020, Article ID 3935762, 18 pages, 2020.

[4] Y. Tian, K. Govindan, and Q. Zhu, "A system dynamics model based on evolutionary game theory for green supply chain management diffusion among Chinese manufacturers," Journal of Cleaner Production, vol. 80, pp. 96-105, 2014.

[5] Q. H. Zhu and Y. J. Dou, "Evolutionary model between governments and core-enterprises in green supply chains," Systems Engineering-Theory \& Practice, vol. 27, no. 12, pp. 85-89, 2007.

[6] X. Liu, K. Lin, L. Wang, and L. Ding, "Pricing decisions for a sustainable supply chain in the presence of potential strategic customers," Sustainability, vol. 12, no. 4, p. 1655, 2020.

[7] D. Ghosh and J. Shah, "A comparative analysis of greening policies across supply chain structures," International Journal of Production Economics, vol. 135, no. 2, pp. 568-583, 2012.

[8] B. Li, M. Zhu, Y. Jiang, and Z. Li, "Pricing policies of a competitive dual-channel green supply chain," Journal of Cleaner Production, vol. 112, no. Part 3, pp. 2029-2042, 2016.

[9] K. Chitra, "In search of the green consumers: a perceptual study," Journal of Services Research, vol. 7, no. 1, pp. 173-291, 2007.

[10] L. Zhang, J. Wang, and J. You, "Consumer environmental awareness and channel coordination with two substitutable products," European Journal of Operational Research, vol. 241, no. 1, pp. 63-73, 2015.

[11] D. Ghosh, J. Shah, and S. Swami, "Product greening and pricing strategies of firms under green sensitive consumer demand and environmental regulations," Annals of Operations Research, vol. 290, no. 1-2, pp. 491-520, 2020.

[12] J. Wen and Y. Li, "Profit distribution model of green building supply chain with fairness preferences and cap-and-trade policy," IOP Conference Series: Earth and Environmental Science, vol. 237, Article ID 052043, 2019.

[13] J.-Y. Chen, S. Dimitrov, and H. Pun, "The impact of government subsidy on supply chains' sustainability innovation," Omega, vol. 86, pp. 42-58, 2019.

[14] Z. Huang, G. Liao, and Z. Li, "Loaning scale and government subsidy for promoting green innovation," Technological Forecasting and Social Change, vol. 144, pp. 148-156, 2019.

[15] M. Franco, H. Haase, and A. Pereira, "Empirical study about the role of social networks in SME performance," Journal of Systems and Information Technology, vol. 18, no. 4, pp. 383403, 2016.

[16] F.-M. Hsu, D.-J. Horng, and C.-C. Hsueh, "The effect of government-sponsored R \& D programmes on additionality in recipient firms in Taiwan," Technovation, vol. 29, no. 3, pp. 204-217, 2009.

[17] W. Wang, Y. Zhang, Y. Li, X. Zhao, and M. Cheng, “Closedloop supply chains under reward-penalty mechanism: retailer collection and asymmetric information," Journal of Cleaner Production, vol. 142, no. Part 4, pp. 3938-3955, 2017.

[18] W. Wang, Y. Zhang, K. Zhang, T. Bai, and J. Shang, "Rewardpenalty mechanism for closed-loop supply chains under responsibility-sharing and different power structures," International Journal of Production Economics, vol. 170, pp. 178-190, 2015.

[19] S. Zhang, Y. Yu, Q. Zhu, C. M. Qiu, and A. Tian, "Green innovation mode under carbon tax and innovation subsidy: an evolutionary game analysis for portfolio policies," Sustainability, vol. 12, no. 4, p. 1385, 2020.

[20] X. Deng, "The incentive effect of modes of government subsidies on technological innovation of LED lighting enterprises in China," Light \& Engineering, vol. 24, no. 3, pp. 145-148, 2016.

[21] S. R. Madani and M. Rasti-Barzoki, "Sustainable supply chain management with pricing, greening and governmental tariffs 
determining strategies: a game-theoretic approach," Computers \& Industrial Engineering, vol. 105, pp. 287-298, 2017.

[22] D. Yang and T. Xiao, "Pricing and green level decisions of a green supply chain with governmental interventions under fuzzy uncertainties," Journal of Cleaner Production, vol. 149, pp. 1174-1187, 2017.

[23] S. Zhan, Z. Shu, and H. Jiang, "Research on two-echelon green supply chain decision under government subsidy," American Journal of Industrial and Business Management, vol. 8, no. 3, pp. 487-495, 2018.

[24] A. Xu and Z. Zhou, "A pricing model for governments' subsidy in the green supply chain," International Journal of Networking and Virtual Organisations, vol. 14, no. 1/2, p. 40, 2014.

[25] Z. Liu, T. D. Anderson, and J. M. Cruz, "Consumer environmental awareness and competition in two-stage supply chains," European Journal of Operational Research, vol. 218, no. 3, pp. 602-613, 2012.

[26] W. Zhu and Y. He, "Green product design in supply chains under competition," European Journal of Operational Research, vol. 258, no. 1, pp. 165-180, 2017.

[27] S. Swami and J. Shah, "Channel coordination in green supply chain management," Journal of the Operational Research Society, vol. 64, no. 3, pp. 336-351, 2013.

[28] W. Zhou, J. Zhou, T. Chu, and H. Li, "A dynamic duopoly cournot model with R \& D efforts and its dynamic behavior analysis," Complexity, vol. 2020, Article ID 4580415, 19 pages, 2020.

[29] L. Zhou, J. Zhou, T. Chu, and H. Li, "Dynamic positioning in ice," Encyclopedia of Ocean Engineering, vol. 2020, Article ID 9634878, 8 pages, 2020.

[30] L. Gori, M. Sodini, and L. Fanti, "A nonlinear cournot duopoly with advertising," Chaos, Solitons \& Fractals, vol. 79, pp. 178-190, 2015.

[31] A. Agliari, N. Pecora, and A. Szuz, "Dynamical analysis of cournot oligopoly models: Neimark-Sacker Bifurcation and related mechanisms," in Qualitative Theory of Dynamical Systems, Tools and Applications for Economic ModellingSpringer Proceedings in Complexity, Urbino, Italy, 2016. 